\title{
Experimental studies on the effect of the number of stages on the performance of an electrical submersible pump in two-phase flow conditions
}

\author{
E. Salehi, J. Gamboa \& M. Prado \\ The University of Tulsa Artificial Lift Project (TUALP), USA
}

\begin{abstract}
The objective of this research is to study experimentally the effect of number of stages on the overall performance of a multistage ESP. Therefore, a series of experiments injecting gas at certain stage and monitoring the pressure increment on stages downstream from the injection point are conducted at different inlet pressure, and rotational speed. The experimental result reveals that two-phase performance of a single stage does not directly represent the two-phase ESP overall performance. However, a strong relationship between two-phase stage performance, number of stages and the pump overall performance exists.

Results also show that increasing the number of stages extends the operating region in which every stage equally contributes on the overall pump performance. This region is bracketed by a critical gas quality that also varies with the increase of the number of stages. Thus, the prediction of the number of stage varies depending on the gas quality. At low gas quality the serial pump rule is suitable for practical application purpose. But, this rule may underestimate the number of stages at higher gas quality in which the serial pump rule is no longer applicable.

Keywords: electrical submersible pump, homogeneous rules, surging initiation, velocity triangle.
\end{abstract}

\section{Introduction}

Electrical Submersible Pumps (ESPs) are a type of multistage centrifugal pumps that due to their high efficiency and compact design have been increasingly used 
as an artificial lift method in deep-water offshore fields. They are especially suitable for high productivity wells with low to moderate viscosity. However, because ESPs are centrifugal pumps, fluid properties such as viscosity, surface tension and free gas considerably affect their overall performance. None of these effects is fully understood. Early studies as those published by Lea and Bearden [3] and Cirilo [1] have shown that the head generated by the ESP and its efficiency drops drastically as the gas-liquid ratio increases.

Deterioration of the ESP performance in the presence of free gas is a function of several operating parameters such as pump intake pressure, pump rotational speed, liquid flow rate, fluid properties and the amount of free gas. At a constant intake pressure, rotational speed, and flow rate, the increase of the amount of free gas causes deterioration which begins as a slight drop in the performance with respect to the water performance and ends in an abrupt performance breakdown in stage head that yields a complete blockage known as gas lock condition.

At a constant liquid flow rate and intake pressure, gradually increasing the gas volumetric fraction (GVF) produces a mild deterioration in stage head until the ESP performance suffers a sharp sudden reduction. This critical point, designated as the surging initiation point, can be characterized by fluctuations in the stage head, motor load and flow rate. Severe head deterioration is observed after the surging initiation point which may yield to gas blockage.

Several studies have been carried out to investigate the effect of various parameters on two-phase flow performance of the ESP, however, in all the experimental studies, the performance of one stage has been a representative of pump overall performance.

This study attempts to collect experimental data that shows the effect of stage number on the overall pump performance. The experimental data collected also shows the relationship between the single stage performance and the overall pump performance. The goal is to develop guidelines that help field and design engineers to predict the minimum number of stage required to handle gassy wells.

\section{Literature review}

Inspired by studies in Loss of Coolant Accidents (LOCA) in nuclear industry, Murakami and Minemura [4, 5] performed the first comprehensive study on the effect of entrained gas in centrifugal pumps. Murakami and Minemura [4, 5] demonstrated the pump head is deteriorated continuously as the air volumetric flow is increased.

The first qualitative study on the two-phase performance of ESP pumps was performed by Lea and Bearden [3]. The same trend of deterioration observed by Murakami and Minemura [4,5] in volute-type centrifugal pumps was confirmed by Lea and Bearden [3] in ESPs. Lea and Bearden's [3] experimental results indicated the importance of the intake pressure and liquid flow rate, as well as pump geometry. Lea and Bearden [3] implicitly assumed pump overall performance is the same as pump stage performance. 
Cirilo [1] suggested that pump head deterioration caused by the gas is a consequence of centrifugal force within the pump impeller that causes accumulation of low-density gas closer to the impeller eye. The gas accumulation restricts the area open to flow for high-density water and pushes it towards the impeller periphery, accelerating the liquid phase to leave the stage faster than the gas phase. This affects the discharge velocity triangle reducing the tangential component of the absolute velocity of the liquid. As a consequence, the pump two-phase head is deteriorated and this leads to further gas accumulation until a sharp breakdown is observed in pump two-phase head. Cirilo [1] also suggested that the flow pattern in the impeller channel goes from bubbly flow to churn flow as the stage gas volumetric fraction increases and the interfacial drag force becomes smaller. Cirilo [1] observed that a mixed-type impeller handles higher gas volumetric fractions compare to a radial impeller at a similar operating condition. Likewise, increasing the pump rotational speed improved the capability of the pump to handle gas.

The effect of number of stages in two-phase ESP performance is not clear from Cirilo's [1] experimental results since the underlying phenomenon is not explained nor examined further. However, Cirilo [1] mentioned that the discharge stages handle smaller gas volumetric fractions than the stage at the inlet region, resulting in a better performance with higher number of stages compare to a small number of stages. Pessoa [6] conducted an experimental study on the stage-by-stage performance of a 22-stage mixed-flow-type ESP handling water and air at intake pressures of 100 psig and 200 psig for a rotational speed of $3,200 \mathrm{rpm}$. Pessoa [6] showed that the single-phase performance of all stages under study is very similar with a good agreement with the manufacturer's catalogue. However, the head created under two-phase flow condition by the stages were not equal. The stages at the inlet region developed less head compared to the stages at the discharge region. Figure 1 illustrates the previously mentioned observation by Pessoa [6]. This figure shows the performance curves of stages 4 and 10 for different mass gas flow rates. A dotted line has been added to each of these graphs, which intercept the performance curve at a constant liquid flow rate. The blue dot line indicates the single-phase pressure increment generated by each stage, which is approximately the same
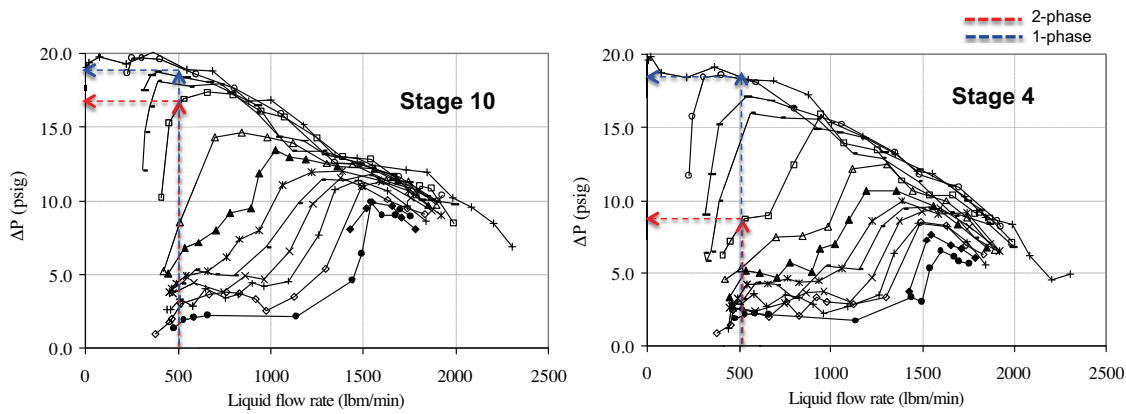

Figure 1: $\quad$ Pressure increment of stage 4 and 10 at different gas flow rates (Pessoa [6]). 
for both stages. The dot red line indicates the stage pressure increment under the two-phase flow for each stage. In this case, the pressure increment of stage 10th is significantly higher than the $4^{\text {th }}$ stage pressure increment. Based on this result, Pessoa [6] concluded that the first stages (located closer to the pump inlet) dissipate most of the energy unlike the other stages that develop head and contribute to the overall pump head.

Gamboa [2] conducted tests at $600 \mathrm{rpm}$ and 2psig intake pressure for several liquid flow rates using a transparent ESP prototype. The experiments were carried out by injecting gas at the intake of the stage and by injecting the gas through a first stage for comparison purpose. The experimental results showed that the stage performance improved when the gas was injected through a previous stage instead of being injected directly at the impeller entrance. Gamboa [2] hypothesized that this effect may be caused by the reduction of the bubble size flowing through the impeller of the investigated stage. Gamboa [2] observed that the bubble size coming out from the first stage was smaller than the one injected directly at the stage. Thus, the first stage pre-conditioned the flow for the next stage.

The mentioned studies are samples of a large number of papers published in the subject, which have investigated the effect of liquid flow rate, intake pressure, rotational speed and impeller geometry. The experimental data collected shows that stage performance differs from each other under two-phase conditions. However, the effect of stage number on the ESP two-phase performance is not well understood.

\subsection{Experimental facility}

A layout of the facility is presented in Figure 2. The loop consists of a $500 \mathrm{bbl}$ open top water storage tank, a set of two pumps (a volute-type centrifugal pump and a progressive cavity pump installed in parallel to provide positive head at the

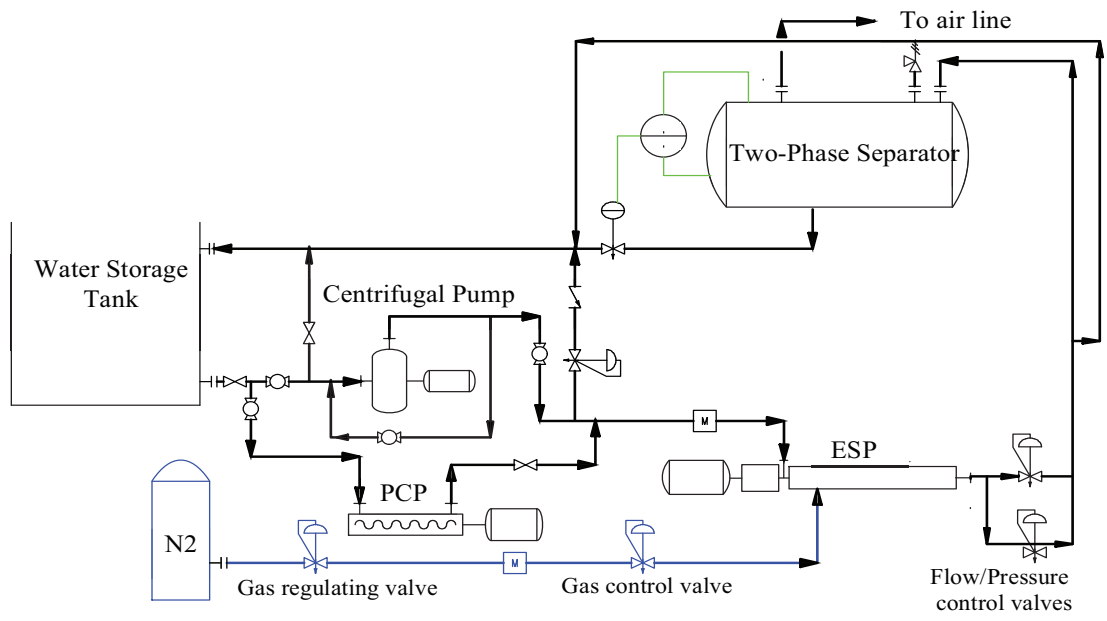

Figure 2: $\quad$ Test facility layout. 
intake of the ESP), the ESP and a two-phase flow separator. Flow rate and intake pressure of the ESP is controlled by two choke valves installed downstream the ESP. Nitrogen is used as the gas phase.

A 14-stage electrical submersible pump with a capacity of 2,700 bpd at the best efficiency point (bep) with specific speed (ns) of 1638 is utilized in this study. This pump has been modified to gather the pressure increment per stage as suggested by Pessoa (2000). Accordingly, a series of quarter-inch (1/4") holes have been drilled on the pump housing at stages $2,3,4,5,6,7,8,9,10,11$ and 12. The modifications previously explained are indicated in Figure 3. Observe Viton O-rings were installed between every stage to avoid fluid communication between stages. Note that the quarter-inch holes were drilled into the lower diffuser of every stage as shown in Figure 3.

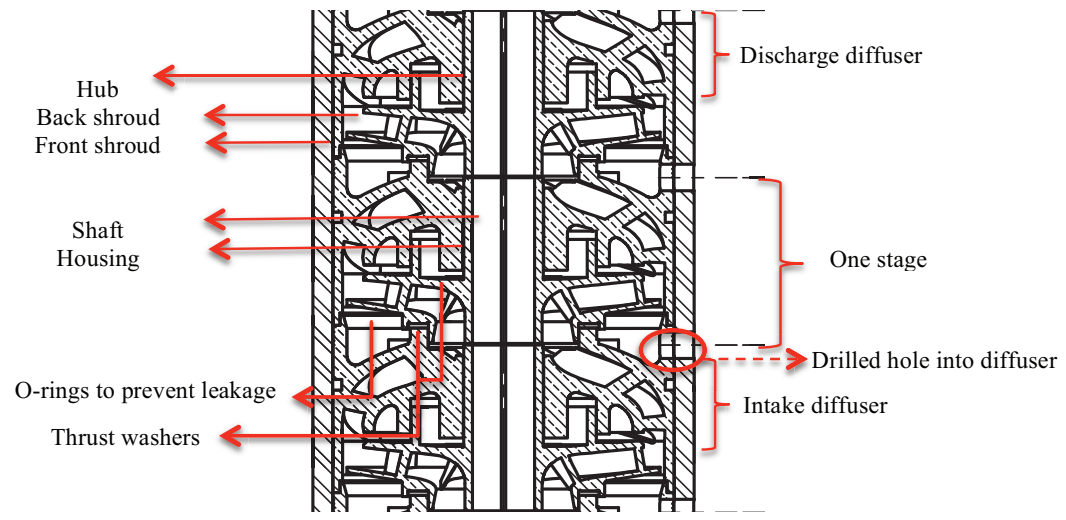

Figure 3: $\quad$ Modified ESP - inside view.

Two differential pressure sensors, three absolute pressure sensors and one temperature sensor are utilized for the measurements. The connection between the differential and absolute pressure sensors and the modified ESP is done through quick-connects and flexible high-pressure nylon hoses as shown in Figure 4.

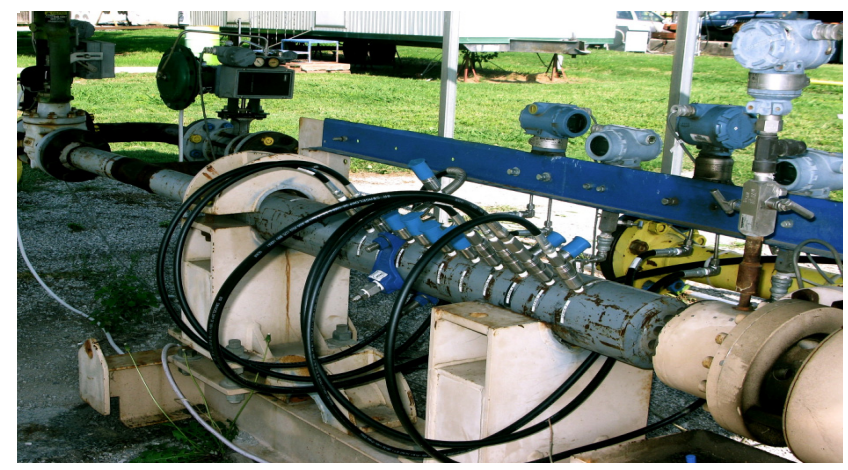

Figure 4: $\quad$ Modified ESP - outside view. 


\subsection{Test fluids}

Tulsa city water and high pressure Nitrogen are used as working fluid in this study. The Tulsa tap water is assumed as incompressible fluid whose chemical composition is mentioned by Urdaneta [7]. Two-parameter Virial EOS is used for calculating the deviation factor and the gas density as function of pressure and temperature.

\subsection{Experimental procedure}

The performance under two-phase conditions is obtained by increasing the mass gas flow rate while the stage intake pressure, rotational speed and liquid flow rate are maintained constant. In order to observe the contribution of every stage on the overall pump pressure increment, the gas is injected at certain stage while the inlet pressure of the previous stage is kept constant at 150 psig. A differential pressure is placed at the first stage downstream from the gas injection stage, while another differential pressure is switched between the other stages located father away from the gas injection point. The gas injection point is varied to reduce or increase the number of stage effectively operating under two-phase flow condition in the same ESP body.

The experiments are carried out at 3,500 rpm for a liquid flow rate of 2700 bpd that corresponds to the bep. The liquid and gas flow rates are measured by Corilios flow meters. Based on the mass flow rate measurements, the gas quality is calculated as ratio of the gas mass flow rate to the gas and liquid mass flow rate.

Results of the tests are presented using the normalized pressure increment defined as:

$$
\Delta P_{n}=\frac{\Delta P_{2 \emptyset}}{\Delta P_{1 \emptyset}}
$$

where $\Delta \mathrm{P}_{1 \emptyset}$ is the single-phase pressure increment and $\Delta \mathrm{P}_{2 \emptyset}$ is the two-phase pressure increment. The single-phase pressure increment is obtained from water performance curve that are presented in the appendix.

\subsection{Results and analysis effect of number of stages in two-phase flow performance}

Figure 5 presents the normalized pressure increment as function of gas quality for stages $4,5,6,7,8,9$ and 10 while the inlet pressure at stage 2 is kept constant at 150 psig. Note that the normalized stage pressure increment reduces with the increase of gas quality in every stage. The performance of all the stages follows a similar trend at gas quality smaller than 0.005 indicating the pressure contribution of every stage on the overall pump performance is the same (pump serial arrangement). The performance of stages 4, 5 and 6 differs from the other stages and each other at gas quality above 0.005 . The difference in performance implies that the pressure contribution of stages 4, 5 and 6 differs from the stages 7, 8, 9 and 10 as mentioned by Pessoa [6]. Severe pressure increment 
deterioration is observed at 0.0012 of gas quality in stage 4, which is also observed in the other stages at higher values of gas quality. At a gas quality of 0.0025 , all the stages approach a normalized pressure increment as low as 0.15 .

Figure 6 shows the normalized pressure increment of the stage 5, 7, 8, 9 and 10 while the gas is injected at the stage 4 and inlet pressure at stage 3 is

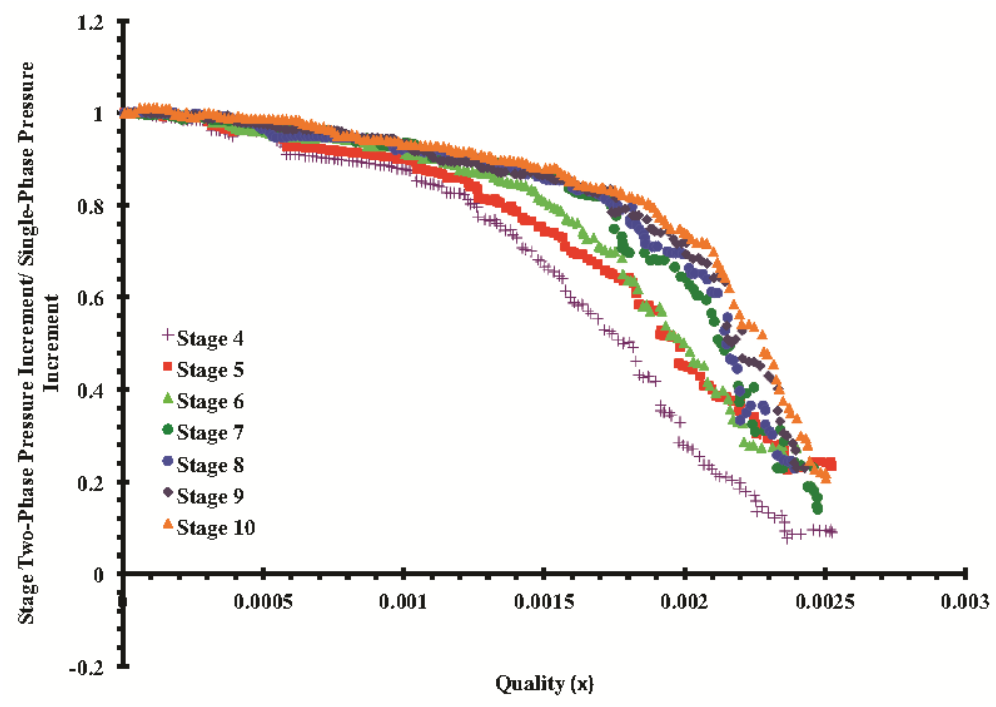

Figure 5: $\quad$ Set 1 test results - gas injection at stage 3 .

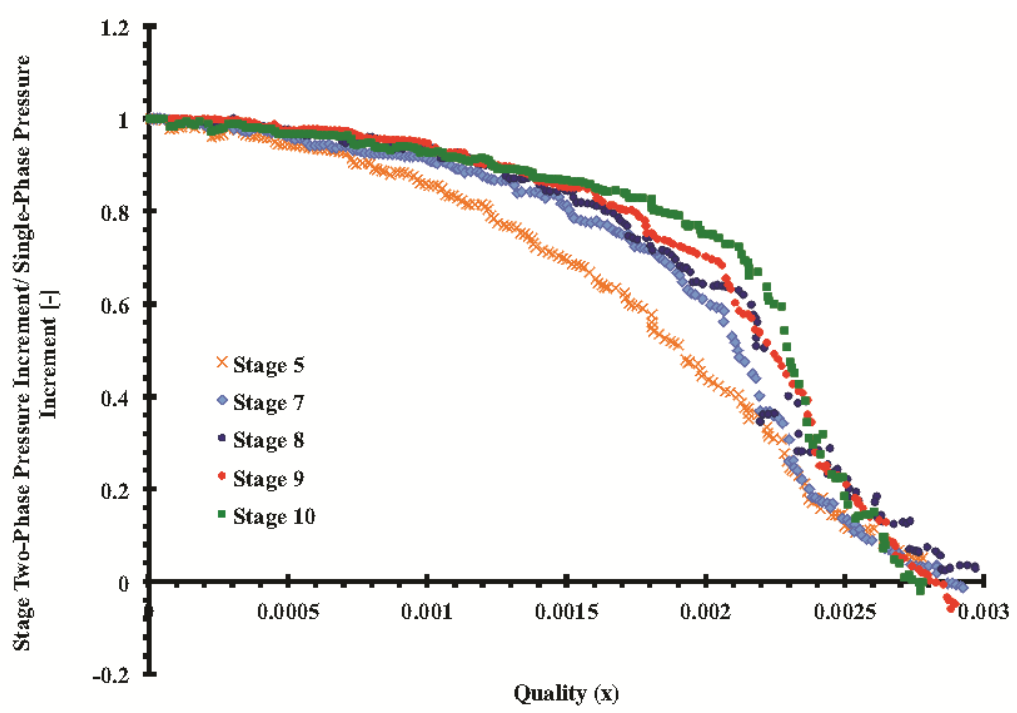

Figure 6: $\quad$ Set 2 test results - gas injection at stage 4 . 
maintained constant at 150 psig. Thus, the effective number of stages under twophase flow is reduced. As mentioned previously, the normalized stage pressure increment reduces continuously with the increase of gas quality. Notice that the normalized pressure increment of all stages are similar at gas quality smaller than 0.001 . Further increase of the gas quality causes that the performance of stage 5 severely deteriorates while the normalized pressure increment is fairly similar. Clear discrepancy between the performance curves is observed at gas quality above 0.0015. At this gas quality the pressure increment of every stage is different. Stage 10 shows that greatest normalized pressure increment while the smallest is observed at stage 5 . At a gas quality of 0.003 , all the stages reach a zero normalized pressure increment.

Figures 5 and 6 demonstrate that at low quality (x) every stage has the same contribution over the overall pressure increment (serial arrangement rule) despite of the gas presence. Further increase of gas quality (x) causes that every stage contributes in different matter on the overall pressure increment.

This effect is further explained in Figure 7. In this figure is shown the twophase stage performance of stages 5, 7 and 10 gathered while the gas is injected at the stage 4 . Observe that three dashed lines have been added to the figure indicating three different operating regions in the two-phase stage performance. The region (1), which ranges from single-phase up to 0.0005 gas quality, all three stages exhibit the same performance that can be depicted as a mild deterioration in stage pressure increment as gas quality is increased. When gas quality has been increased between 0.0005 and 0.0015 , the stage performance reaches region (2). The deterioration becomes more severe in this region; however, the pressure increment developed by stages 7 and 10 is higher

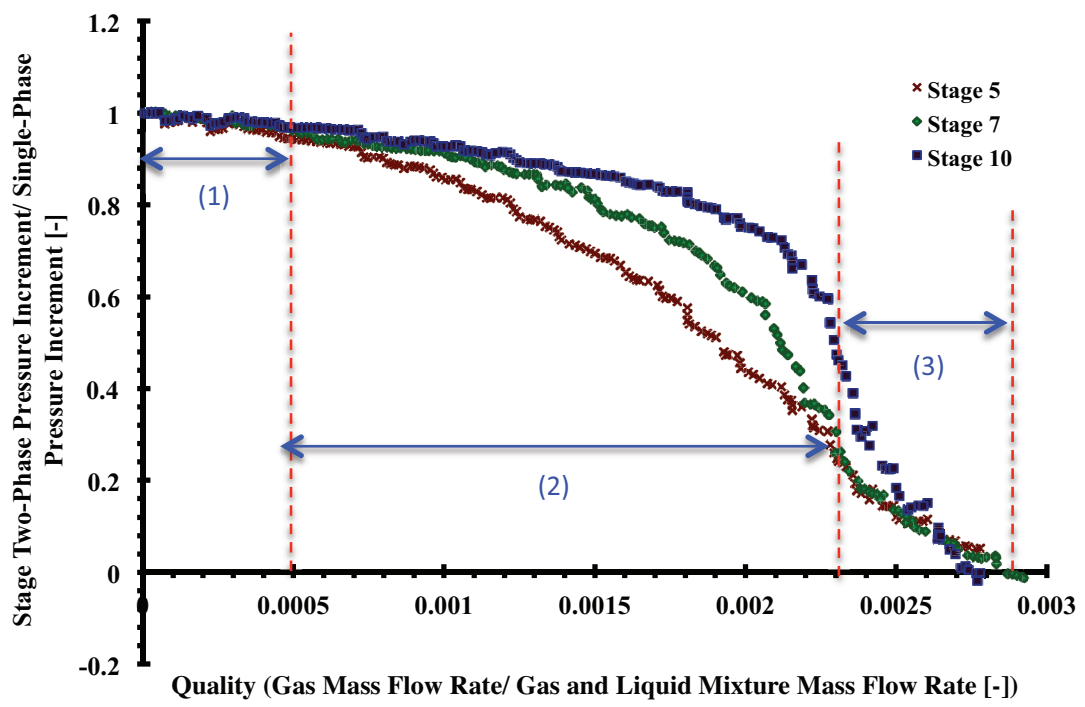

Figure 7: $\quad$ Effect of number of stages at 150 psig, $3500 \mathrm{rpm}$ and $2700 \mathrm{bpd}$ water and N2. 
compared to the pressure increment of stage 5 . At 0.0015 gas quality, the stage 10 pressure increment is $89 \%$ of its single-phase pressure increment while the pressure increment developed by stage 5 is only $77 \%$. The performance curve for stage 7 collapses into the performance curve of stage 5 at gas quality of 0.0023 while the difference between pressure increment of stage 5 and stage 10 reaches a maximum at this gas quality. In region (3) the stage 10 suffers a severe deterioration that causes a very sharp reduction of the pressure increment in less than $5 \%$ of increase in gas quality. The reduction of pressure increment is such that the pressure increment collapses into stage 5 and stage 7 performance curves at 0.003 gas quality.

Observe that stage 10 adds more pressure on the overall pressure increment than stages 5 and 7, which is consistent with Pessoa [6] observations. The performance of last stages is closer to the single-phase performance than the first stages performance demonstrating the existence of flow conditioning in the stages.

Once the severe deterioration has initiated in stage 10, the overall pump pressure increment quickly drops down until it reaches a value close to zero. It implies that there is no stage generating pressure so that one may speculate the overall pump surging initiation coincides with the last stage surging condition.

The experimental data shows the existence of a critical gas quality above which performance of every stage collapses in a single trend. This critical gas quality seems to moves to higher values as the number of stages is increased. This critical condition may be utilized for defining the minimum number of stages required for pre-conditioning the gas and liquid mixture of the discharge stages.

\section{Conclusions}

- $\quad$ The data obtained from the ESP demonstrates the effect of gas ingested by the pump on the stage performance of the ESP.

- As GVF increases, the ESP stage performance deteriorates; the deterioration is very mild until GVF reaches to a critical value where surging initiates. After this point, the rate of deterioration becomes a function of stage number.

- In an interval of quality starting from the quality at which surging initiates at the first stage, the stages closest to the gas injection point suffer head deterioration while the stages beyond this point deteriorate very mildly. As this quality interval ends, the rate of deterioration becomes severe in all the stages specifically last stages.

\section{Appendix}

\section{Single-phase performance}

Single-phase tests are performed in order to compare the actual performance of the pump with the data obtained from the catalogue at full speed, $3500 \mathrm{rpm}$ and 


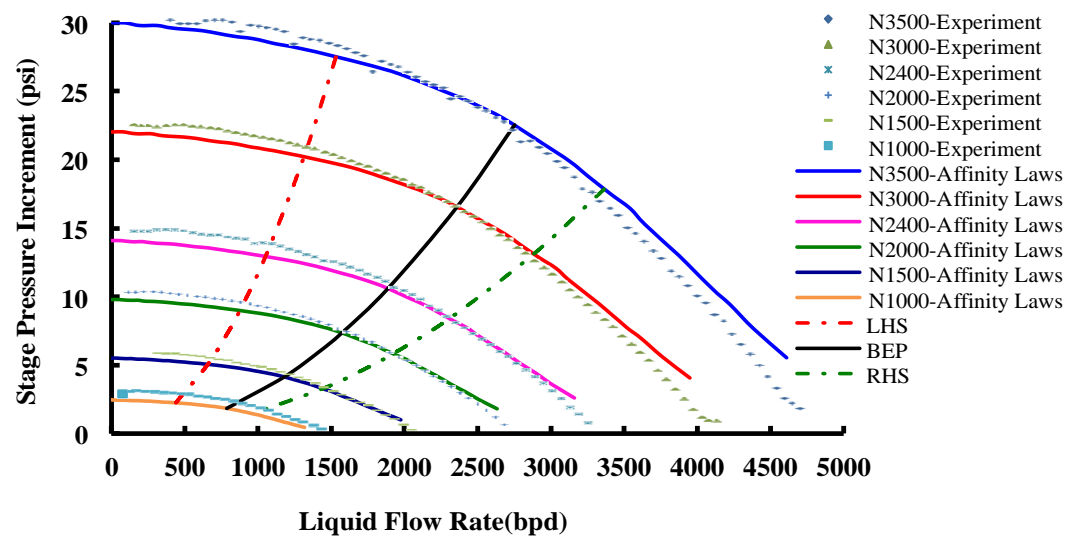

Figure 8: Single-phase stage performance curves at different rotational speed.

the performance curves that are obtained by applying homogeneous laws. Figure 8 presents the performance curve at different rotational speeds under single-phase flow condition using fresh water. $\mathrm{X}$-axis represents the liquid flow rate in barrels per day, and Y-axis is stage 5's pressure increment for one stage in psi. In this figure, the experimental data obtained from the tests are shown by dot points, and the manufacture performance obtained by applying the affinity laws to the pump performance curve at $3500 \mathrm{rpm}$ are indicated by solid lines. The three ascending lines represent left-hand-side (LOR), best efficiency point (BEP) and right-hand-side (ROR) of the pump operating window, respectively. These curves are obtained applying affinity laws to the original curve from the manufacturer catalogue, as well. Figure 8 shows water performance curves are consistent with the curves obtained from affinity laws formula within the pump's operating window. As it is expected from a centrifugal type pump, the stage pressure increment descends as the liquid flow rate increases.

\section{References}

[1] Cirilo, R. Air-Water Flow through Electrical Submersible Pumps. Vol. MS Thesis. Tulsa: The University of Tulsa, 1998.

[2] Gamboa, J. Prediction of the Transition in Two-Phase Performance of an Electrical Submersible Pump. Vol. PhD Dissertation. Tulsa: The University of Tulsa, 2008.

[3] Lea, J. F and Bearden, J. C. "Effect of Gaseous Fluids on Submersible Pump Performance.” Journal of Petroleum Technology 34, no. 12 (1982): 29222930.

[4] Murakami, M., and Minemura, K. "Effects of Entrained Air on the Performance of Centrifugal Pump, 2nd Report, Effect of Number of Blades.” Bulletin of the JSME 17, no. 112 (1974): 1286-1295. 
[5] Murakami, M., Minemura, K. "Effects of Entrained Air on the Performance of a Centrifugal Pump, 1st Report, Performance and Flow Condition.” Bulletin of the JSME 17, no. 110 (1974): 1047-1055.

[6] Pessoa Rodrigues, R. Experimental Investigation on Two-Phase Flow Performance of Electrical Submersible Pump Stages. Tulsa, OK: The University of Tulsa, 2001.

[7] Urdaneta, N. Utilization of Dispersion Characterization Rig (DCR) to Study the Effect of Salinity and Dimulsifiers on Oil-Water Dispersion Separation. Tulsa, OK: The University of Tulsa, 2009. 